\title{
Proposed Multi-Modal Palm Veins-Face Biometric Authentication
}

\author{
S.F.Bahgat \\ College of computers and IT \\ Taif University \\ Taif, KSA
}

\author{
S. Ghoniemy \\ College of computers and IT \\ Taif University \\ Taif, KSA
}

\author{
M. Alotaibi \\ College of computers and IT \\ Taif University \\ Taif, KSA
}

\begin{abstract}
Biometric authentication technology identifies people by their unique biological information. An account holder's body characteristics or behaviors are registered in a database and then compared with others who may try to access that account to see if the attempt is legitimate. Since veins are internal to the human body, its information is hard to duplicate. Compared with a finger or the back of a hand, a palm has a broader and more complicated vascular pattern and thus contains a wealth of differentiating features for personal identification. However, a single biometric is not sufficient to meet the variety of requirements, including matching performance imposed by several large-scale authentication systems. Multi-modal biometric systems seek to alleviate some of the drawbacks encountered by uni-modal biometric systems by consolidating the evidence presented by multiple biometric traits/sources. This paper proposes a multi-modal authentication technique based on Palm Veins as a personal identifying factor, augmented by face features to increase the accuracy of security recognition. The obtained results point at an increased authentication accuracy.
\end{abstract}

Keywords-Biometric authentication; Face Recognition; Feature Fusion; Palm veins; Statistical features.

\section{INTRODUCTION}

Biometrics is automated methods of recognizing a person based on a physiological or behavioral characteristic. Among the features measured are: face, fingerprints, hand geometry, iris, retinal, veins, handwriting, gait, and voice. Biometric systems are superior because they provide a nontransferable means of identifying people, not just cards or badges. The key point about an identification method that is "nontransferable" means it cannot be given or lent to another individual, so nobody can get around the system - they personally have to go through the control point.

A key advantage of biometric authentication is that biometric data is based on physical characteristics that stay constant throughout one's lifetime and are difficult to fake or change. Fingerprints, palm vein, and iris scans can produce absolutely unique data sets when done properly. It is not easy to determine which method of biometric data gathering and reading does the "best" job of ensuring secure authentication. Each of the different methods has inherent advantages and disadvantages [1].

Palm vein authentication uses an infrared beam to penetrate the users hand as it is held over the sensor; the veins within the palm of the user are returned as gray lines. As each Biometrics technology has its merits and shortcomings, it is difficult to make direct comparisons, but because vein authentication relies on biological information on the interior of the body, it is more effective than the others at reducing the possibility of falsification. Also, vein pattern recognition requires just a scan of the palm, thus making it the easiest and most natural to use among the various biometric technologies [2].

Moreover, to confirm the accuracy of personal authentication to an even greater degree, vein recognition can be combined with face recognition systems to enable "multimodal authentication" that guarantees accuracy through multiple layers of security. In addition to enhanced security, vein authentication used in conjunction with face recognition systems would also keep a log of facial information should it be necessary to be used as evidence [3].

This paper proposes a bi-modal biometric authentication system that fuses the features of the palm veins with that of the face for increasing authentication accuracy. At first, 4 types of statistical features are tested for the best recognition accuracy for palm veins and face independently. The feature sets best performing for each biometric are fed to a feature fusion strategy for increased authentication accuracy.

The rest of the paper is organized as follows. Section 2 scans the previous work in the related areas. Section 3 presents the structure of the proposed system. Section 4 analyzes the obtained results. The paper is terminated by a conclusion summarizing the obtained results and specifying problems for future work.

\section{PREVIOUS WORKS}

Ishani Sarkar et al [4] presented a review on the palm vein authentication device that uses blood vessel patterns as a personal identifying factor. As biometric technology matures, there will be an increasing interaction among the market, technology, and the applications. This interaction will be influenced by the added value of the technology, user acceptance, and the credibility of the service provider. It is too early to predict where and how biometric technology would evolve and get embedded in which applications. But it is certain that biometric-based recognition will have a profound influence on the way we conduct our daily business.

Masaki Watanabe et al [2], have shown a biometric authentication using contactless palm vein authentication device that uses blood vessel patterns as a personal identifying factor. Implementation of these contactless identification systems enables applications in public places or in 


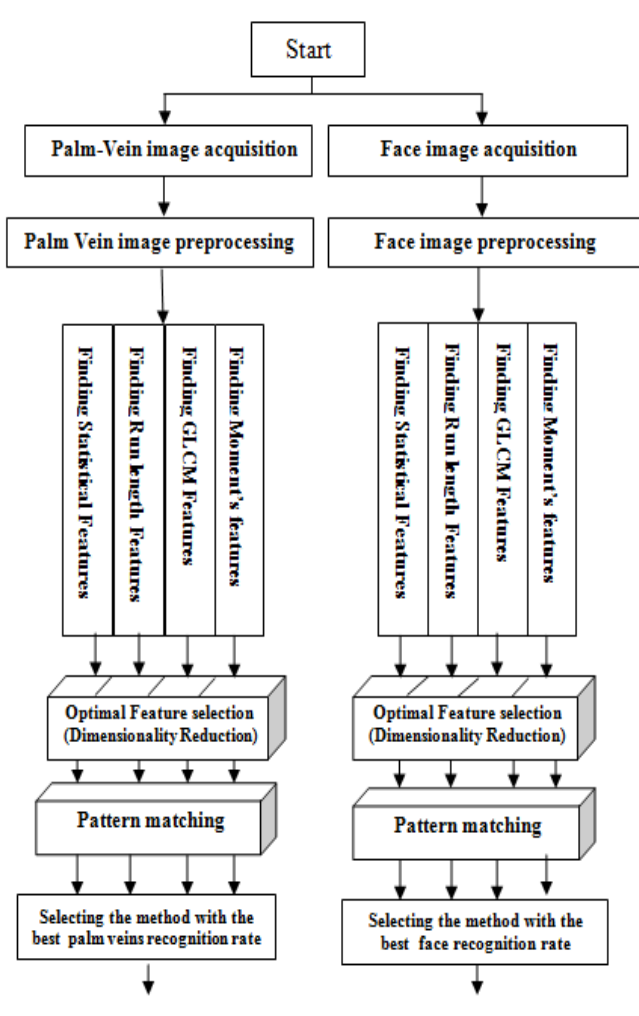

Fig.1 Flow of processes of the preparation phase

environments where hygiene standards are required, such as in medical applications. In addition, sufficient consideration was given to individuals who are reluctant to come into direct contact with publicly used devices.

Yingbo Zhou and Ajay Kumar [5] presented two palm vein representations, using Hessian phase information from the enhanced vascular patterns in the normalized images, and secondly from the orientation encoding of palm vein line-like patterns using localized Radon transform. The experimental results suggest that the proposed representation using localized Radon transform achieves better or similar performance than other alternatives while offering significant computational advantage for online applications. The proposed approach achieves the best equal error rate of $0.28 \%$. Finally, they proposed a score level combination strategy to combine the multiple palm vein representations, thus achieved consistent improvement in the performance, both from the authentication and recognition experiments, which illustrates the robustness of the proposed schemes.

Yi-Bo Zhang et al [6] proposed a scheme of personal authentication using palm vein. The proposed system includes: 1) Infrared palm images capture; 2) Detection of Region of Interest; 3) Palm vein extraction by multiscale filtering; 4) Matching. The experimental results demonstrate that the recognition rate of their system is fine but not good enough to be a real system. The capture device is very sensitive to the outside lights. The outside lights can affect the inside infrared light source so that some images have very poor quality. If the capture device can be improved, the system performance should be better. Further, the database is too small to be convincible. More data are required to be collected for the evaluation of the system.

Alaa ELEYAN, and Hasan DEM'IREL [7], introduced a new face recognition technique based on the gray-level cooccurrence matrix (GLCM). GLCM represents the distributions of the intensities and the information about relative positions of neighboring pixels of an image. They proposed two methods to extract feature vectors using GLCM for face classification. The first method extracts the wellknown Haralick features from the GLCM, and the second method directly uses GLCM by converting the matrix into a vector that can be used in the classification process. The results demonstrate that the second method, which uses GLCM directly, is superior to the first method that uses the feature vector containing the statistical Haralick features in both nearest neighbor and neural networks classifiers. The proposed GLCM based face recognition system not only outperforms well-known techniques such as principal component analysis and linear discriminant analysis, but also has comparable performance with local binary patterns and Gabor wavelets. It is obvious from the results that the GLCM is a robust method for face recognition with competitive performance.

Muhammad Imran Razzak et al [3], presented multimodal face and finger veins recognition systems in which multilevel score level fusion was performed. Since there is no database for finger veins and face, thus they test the CAIRO employer and students. The imposter and genuine score are combined using Fuzzy fusion to increase the face recognition system. Simulation results shows that proposed multimodal recognition system is very efficient in reducing the FAR 0.05 and increasing GAR 91.4. The GAR and FAR can further be optimized by applying class to client approach on finger veins.

\section{PROPOSED SYSTEM}

Due to the increase in security requirements, biometric systems have been commonly utilized in many recognition applications. Multimodal systems have great demands to overcome the issue involved in single trait systems and this has become one of the most important research areas of pattern recognition. This paper presents a multimodal palm veins and face biometric verification system to improve the performance that fuses palm veins and face features for better authentication accuracy. The proposed system proceeds as follows (for both biometric systems (palm veins and face):

\section{A. Preparation Phase:}

In this phase, we estimate the performance of four statistical feature extraction methods and adopt the one with the best recognition rate. The process proceeds as shown in Fig. 1.

\section{1) Image Acquisition:}

The palm vein images were captured using "M2PalmVein ${ }^{\mathrm{TM}}$ Reader" [8], for 18 persons.

The face images were captured using ........for the same 18 persons; examples of the captured images are shown in Figure 2. 


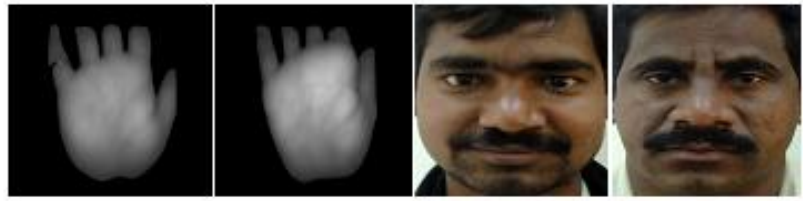

Figure 2 Examples of the captured palm veins and face images

2) Preprocessing:

As the quality of the palm veins images were very bad, several preprocessing techniques were used to enhance the image quality. On the other hand, the facial images were noise-cleaned and contrast enhanced. An example of the enhanced images is shown in Figure 3.

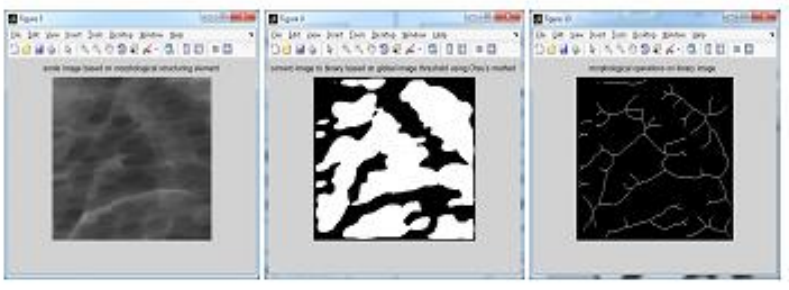

Fig. 3 Examples of palm veins images after preprocessing

3) Feature selection

Four statistical approaches are applied for feature extraction; namely: Gray-level Co-occurrence Matrix (GLCM), Run-Length Matrix (RLM), Statistical Features (SF), and Moment Invariants (MIs). The paper aims at selecting the best performing approach for both biometrics. For that, we distorted the original images with three different types of noise; each with different noise levels. Salt and pepper, impulse and Gaussian noise types with intensity of $5 \%, 10 \%$, and $15 \%$ are used throughout the study. The used feature selection techniques are as follows:

1) Statistical features The following set of features is used [9]:

"Mean, Variance, Smoothness, Skewness,

Kurtosis, Uniformity, and Entropy"

2) Gray-level-Concurrence Matrix (GLCM) The following set of features is used [10]:

"Homogeneity (Angular Second Moment (ASM)), Energy, Entropy, Means, Variance, and Correlation"

3) Run-length matrix (RLM) The following set of features is used [11]:

"Short run emphasis (SRE), Long run emphasis (LRE), High gray-level run emphasis (HGRE), Low gray-level run emphasis (LGRE), Pair-wise combinations of the length and gray level emphasis (SRLGE, SRHGE, LRLGE, LRHGE), Run-length non-uniformity (RLNU), Grey-level nonuniformity (GLNU), and Run percentage (RPC)"

4) Moment Invariants (MIs) [12,13]:

Most frequently used are the $\mathrm{Hu}$ set of invariant moments which are invariant under translation, changes in scale, and also rotation.

5) Dimensionality reduction [14]:
To avoid the curse of dimensionality, PCA is applied by projecting the data onto a lower-dimensional space. This technique is applied for the feature vectors of the four feature extraction approaches.

6) Selecting the best-performing approach:

The recognition rate of each approach is determined using ten data sets; the original (clean) data set, and nine corrupted data sets with different types of noise with different noise levels. The average recognition rate is calculated and the best performing feature set is adopted for post processing.

7) The best approach for each biometric is selected.

\section{B. Training Phase}

The training phase algorithm is depicted in Fig. 4. In this phase:

- The palm veins and face images are captured in the same way as in the preparation phase

- The captured images pass through the same preprocessing stages.

- The feature vectors are extracted using the technique selected in the preparation phase.

- PCA is applied on both feature vectors for dimensionality reduction.

- The sets of features of both biometrics are fused.

- The fused feature vector is stored in database for future comparisons.

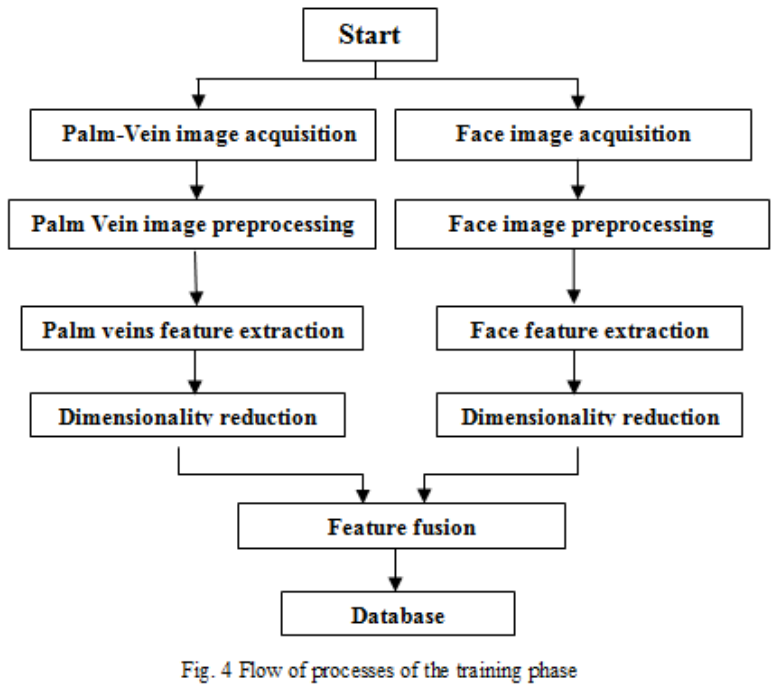

\section{Testing Phase}

- The images of the person under test are acquired and preprocessed typically as in the preparation phase.

- The feature vector of the adopted approach is calculated and reduced using PCA.

- The resulting feature vector is compared with those stored in database and the person is recognized.

The testing phase algorithm is depicted in Fig. 5 


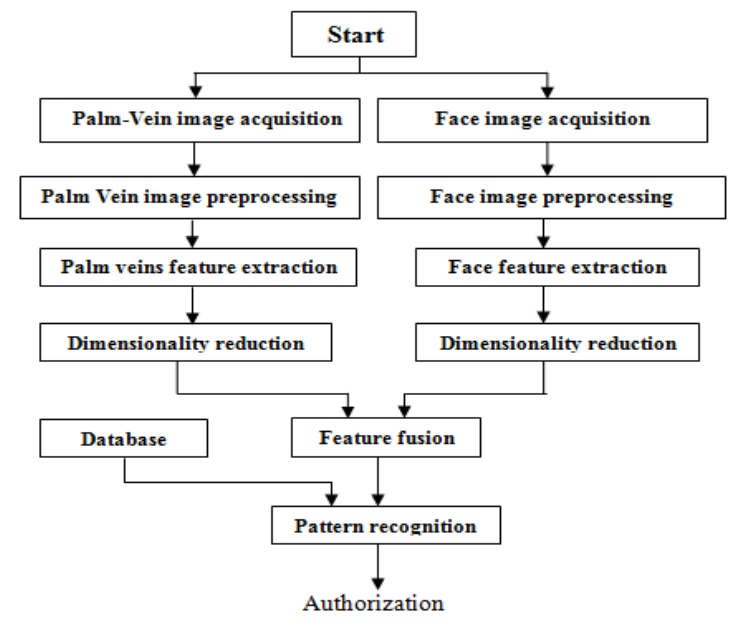

Fig. 5 Flow of processes of the testing phase

\section{Results And Discussion}

For palm veins, and according to Fig. 6, it is clear that the Moment Invariants method gives the best recognition rate for all types of noise. However, for face; and according to Fig. 7, both GLCM and Moment Invariants give comparable recognition rates. As the GLCM requires more computations, we adopted Moment Invariants feature vectors for both biometrics.

Table 1 Average Palm-veins Recognition rate for different noise types

\begin{tabular}{|l|c|c|c|}
\hline & salt \& pepper & impulse noise & Gaussian \\
\hline Statistical & 29.16666667 & 31.944444444 & 29.16666667 \\
\hline RLM & 29.16666667 & 29.16666667 & 29.16666667 \\
\hline GLCM & 36.11111111 & 36.11111111 & 29.16666667 \\
\hline Moment & 72.22222222 & 77.77777778 & 72.22222222 \\
\hline
\end{tabular}

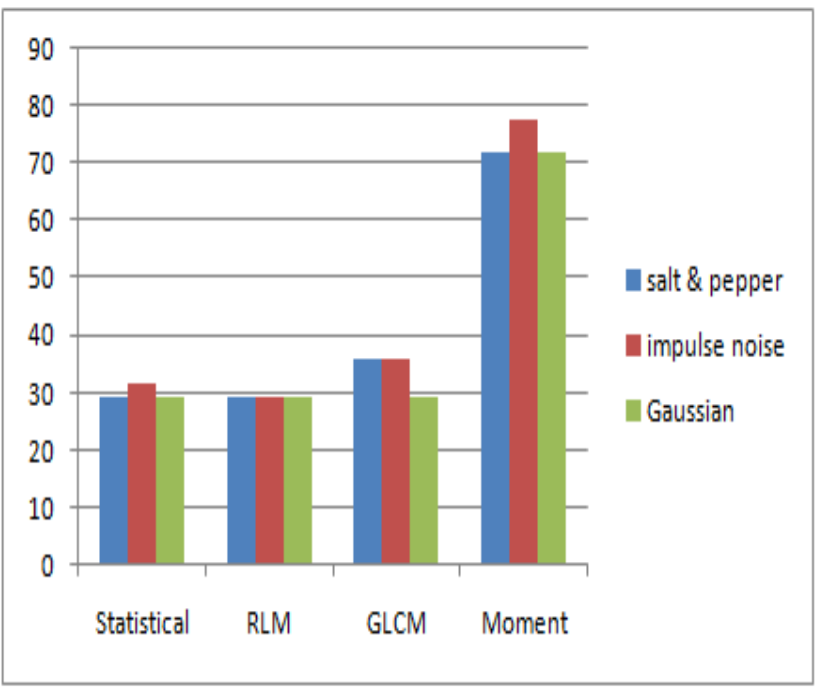

Fig. 6 Average Palm-veins Recognition rate for different noise types
Table 2 Average face Recognition rate for different noise types

\begin{tabular}{|l|c|c|c|}
\hline & salt \& pepper & impulse noise & Gaussian \\
\hline RLM & 63.88889 & 58.33333 & 29.16667 \\
\hline GLCM & 70.83333 & 69.44444 & 29.16667 \\
\hline Moment & 66.66667 & 70.83333 & 30.55556 \\
\hline Statistical & 40.27777778 & 36.11111111 & 29.16667 \\
\hline
\end{tabular}

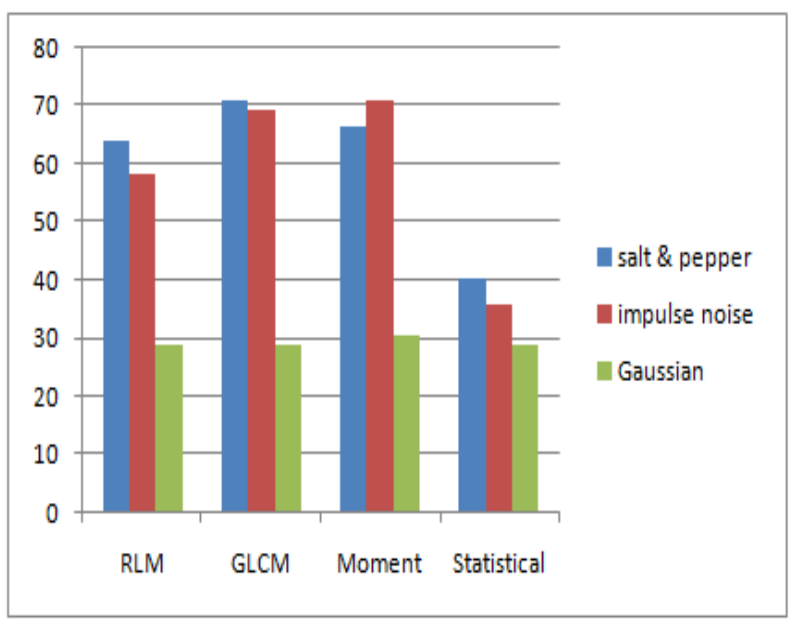

Fig. 7 Average Face Recognition rate for different noise types

For evaluating the performance of the proposed system, we calculated the recognition rate for palm veins images corrupted by salt \& pepper noise with intensities of $0,15,20$, and $25 \%$ respectively. The obtained results are shown in Table 3 . Simultaneously, we calculated the recognition rate using the fused feature vector for the same type of noise with the same intensity levels. The results are shown in Table 4 . It is clear that an average of $30 \%$ enhancement in the recognition rate is obtained, Fig. 8 . 
Table 3 Recognition rate of images comupted by salt \& pepper noise with differe intensities using palm veins and fused feature vectors respectively

\begin{tabular}{|c|c|c}
\hline Noise Intensity & $\begin{array}{c}\text { Recognition rate using } \\
\text { palm veins feature vector }\end{array}$ & $\begin{array}{c}\text { Recognition rate using } \\
\text { fused feature vector }\end{array}$ \\
\hline $0 \%$ & $100 \%$ & $100 \%$ \\
\hline $15 \%$ & $84.444 \%$ & $100 \%$ \\
\hline $20 \%$ & $66.66 \%$ & $94.44 \%$ \\
\hline $25 \%$ & $44.44 \%$ & $90.44 \%$ \\
\hline average & $73.882 \%$ & $96.22 \%$ \\
\hline
\end{tabular}

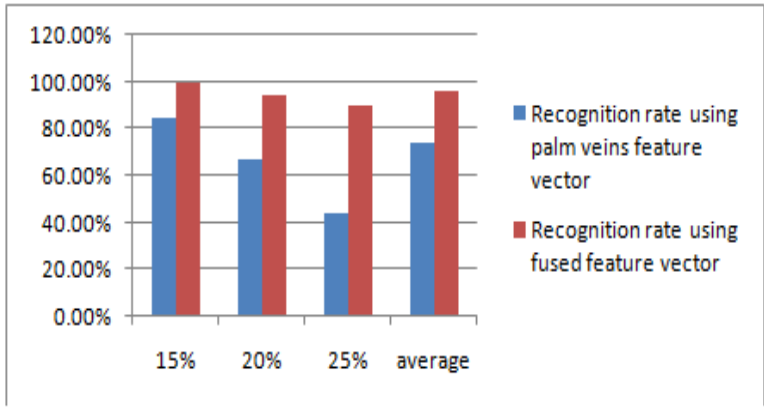

Fig. 8 Recognition rate enhancement usig feature fusion

\section{CONCLUSIONS ANd FUtURE WORK}

This paper studies the contribution of four different statistical approaches in recognizing persons through their palm veins and face images. These approaches are Gray-level Co-occurrence Matrix (GLCM), Run-Length Matrix (RLM), Statistical Features (SF), and Moment Invariants (MIs). The images under consideration are corrupted by different types of noise with different noise intensities, and the recognition rate is evaluated in each case. It was found that Moment Invariant (MIs) feature vector guarantees the best recognition rate in all cases. The MIs are then fused and applied for estimating the resulting recognition rate. It was found that this fusion enhances the recognition rate by more than $30 \%$. Further analysis is required for more enhancements.

\section{References}

[1] Bhudev Sharma, "Palm Vein Technology", Technical Report, Electronics Engineering Department, National Institute of Technology, India, 2010.

[2] Masaki Watanabe, Toshio Endoh,Morito Shiohara, and Shigeru Sasaki," Palm vein authentication technology and its applications", The Biometric Consortium Conference, September 19-21, 2005,USA.

[3] Muhammad Imran Razzak, Rubiyah Yusof and Marzuki Khalid, "Multimodal face and finger veins biometric authentication", Scientific Research and Essays Vol. 5(17), pp. 2529-2534, ISSN 1992-2248 (C)2010 Academic Journals.4 September, 2010.

[4] Ishani Sarkar, Farkhod Alisherov, Tai-hoon Kim, and Debnath Bhattacharyya, "Palm Vein Authentication System: A Review", International Journal of Control and Automation, Vol. 3, No. 1, March, 2010.

[5] Yingbo Zhou, Ajay Kumar, "Contactless Palm Vein Identification using Multiple Representations", Department of Computing, The Hong Kong Polytechnic University.

[6] Yi-Bo Zhang, Qin Li, Jane You, and Prabir Bhattacharya "Palm Vein Extraction and Matching for Personal Authentication", Biometrics Research Centre, Department of Computing, Concordia University,
Quebec, Canada, Spring - Verlag Berlin Heidelerg 2007. http://www.academicjournals.org/SRE

[7] Alaa ELEYAN, Hasan DEM'IREL, "Co-occurrence matrix and its statistical features as a new approach for face recognition”, Turk J Elec Eng \& Comp Sci, Vol.19, No.1, 2011.

[8] http://www.m2sys.com/pdf/M2-PalmVein.pdf

[9] Fazal Malik and Baharum Baharudin, "The Statistical Quantized Histogram Texture Features Analysis for Image Retrieval Based on Median and Laplacian Filters in the DCT Domain”, IAJIT First Online Publication, 2012.

[10] Fritz Albregtsen, "Statistical Texture Measures Computed from Gray Level Coocurrence Matrices," International Journal of Computer Applications, November 5, 2008.

[11] Xiaoou Tang, "Texture Information in Run-Length Matrices", IEEE Transactions on Image Processing, Vol. 7, No. 11, November 1998.

[12] Z. Song, B. Zhao, Z. Zhu, E. Mao "Research on Traffic Number Recognition Based on Neural Network and Invariant Moments", Proceedings of the Sixth International Conference on Machine Learning and Cybernetics, Hong Kong, 19-22 August 2007.

[13] Zhihu Huang, Jinsong Leng, "Analysis of Hu's Moment Invariants on Image Scaling and Rotation", $20102^{\text {nd }}$ International Conference on Computer Engineering and Technology, vol 7 pp 476-480, 2010.

[14] Aamir Khan and Hasan Farooq, "Principal Component Analysis -Linear Discriminant Analysis Feature Extractor for Pattern Recognition”, IJCSI International Journal of Computer Science Issues, Vol. 8, Issue 6, No 2, November 2011, ISSN (Online): 1694-0814. 